\title{
Heterosis Studies in Indian Mustard [Brassica juncea (L.) Czern \& Coss.] for Yield and its Related Attributes
}

\author{
H. V. S. Shekhawat ${ }^{1 *}$, S. S. Shekhawat ${ }^{2}$ and U. S. Shekhawat ${ }^{3}$ \\ ${ }^{1}$ Department of Plant Breeding \& Genetics, AU, Jodhpur, India \\ ${ }^{2}$ Department of Plant Breeding \& Genetics, SKRAU, Bikaner, India \\ ${ }^{3}$ FormerProfessor and Zonal Director of Research (ZDR), ARS, Sri Ganganagar, India \\ *Corresponding author
}

\section{A B S T R A C T}

Keywords

Environment, Standard Heterosis, Pooled Data, Crosses

Article Info

Accepted:

15 December 2020 Available Online: 10 January 2021
Above titled experiment was conducted at two locations, College of Agriculture, Bikaner and ARS, Sri Ganganagar respectively. In this experiment, $125 \mathrm{~F}_{1} \mathrm{~s}, 25$ parent genotypes (20 lines and 5 testers) and one check were analyzed at each location. For the study, a total of four environments were created, each location had two environments, timely sown and late sown, respectively. Subsequently, on the basis of pooled data of four environments standard heterosis was estimated for twelve yield and yield related attributes over the check variety RGN-13. Through the study, significant standard heterosis results were obtained for most of the traits including seed yield per plant. From the performance point of view, crosses RGN 73 x Kranti, Vardan x Kranti; Vardan x RB-50 and; RH-749 x RB50 reported significant standard heterosis for many characters. For almost all the characters variable number of crosses depicted standard heterosis in both positive and negative direction, indicating that genes with negative as well as positive effects were dominant. This show of unpredictability highlights the role of non additive gene actions, which in turn may be due to dominance and/or epistasis.

\section{Introduction}

The oilseeds occupy significant place in Indian economy next to food grain. India is the third largest producer of oilseeds in the world. Oilseed Brassicas are the second most important oilseed crops of the world after Soybean (Anonymous, 2015). India, China, Canada, Japan and European Union are the major Brassica growing regions of the world (Anonymous, 2016).
The mustard is grown not only as oilseed crop but also as condiments and for medicinal use. The oil content in mustard seed ranges from 30$42 \%$. Its leaves and tender stem form the most popular winter greens in some Indian states. Mustard oil has peculiar pungency due to the presence of sulphur compound, thus making it suitable for condiments and for preparation of pickles, curries and vegetables. Its leaves are rich in protein, minerals and vitamin $\mathrm{A}$ and $\mathrm{C}$. After extraction of oil from seeds, the remnant is used as food for milching cattle. 
The mustard is cultivated during the rabi season in India and requires relatively cool temperature, a fair supply of soil moisture during the growing period and a dry weather during the harvest period. The plant can tolerant wide range of soil $\mathrm{pH}$ and can be successfully grown in light sandy to marginal soils, though light loam to heavy loam soils are the best suited. This crop is grown both under rainfed as well as irrigated conditions either as a pure crop or as a mixed crop.

To cope up with the increasing population and demand of oilseeds, various strategies have been evolved to improve the yield levels in the country that are far below the world levels. The systematic genetic improvement efforts have resulted into release of about 158 varieties. Further, taking the advantage of recently available CMS system in addition to barnasebarstar system six hybrids have also been developed.

However, yield realization falls much behind the world average and need genetic interventions to further improve the potential of the varieties and hybrids. In this background the present study is justifiable and necessary timely effort. However, in future available male sterile lines may be involved allowing direct exploitation of heterosis at commercial level. The identified hybrid combinations for high heterosis may be tested again with larger plot size to determine their potential for commercial exploitation.

\section{Materials and Methods}

The present investigation was carried out at Research farm of College of Agriculture, Beechwal, Bikaner (Zone Ic) and at ARS, Sri Ganganagar (Zone Ib) during 2015-16 Rabi season. The experimental material consisted of 25 lines, 5 testers, their 125 cross combinations and one check. The crosses for the study were developed at ARS, Sri Ganganagar during 2014-15 Rabi season through Line $x$ Tester mating design. During 2015-16 Rabi season all the genotypes (crosses, parents and check) were sown in each of the four environments in randomized block design with three replications. Each genotype was sown in single row plot of $3 \mathrm{~m}$ row length. Row to row and plant to plant distances were maintained at $45 \mathrm{~cm}$ and 15 $\mathrm{cm}$ respectively, in each replication. Recommended package of practices were adopted to raise a good crop.

The characters studied were: days to $50 \%$ flowering, days to maturity, plant height, number of primary branches per plant, number of siliqua per plant, siliqua length, number of seeds per siliqua, 100-seed weight, stover yield per plant, seed yield per plant, harvest index and oil content.

\section{Results and Discussion}

In the study, standard heterosis was estimated over the check variety RGN-13.In matters of performance for different traits various crosses were found to be giving top performances viz, RH-749 x RB-50, RGN$236 \times$ RGN-145 and RGN-236 x RB-50 were identified as highly heterotic crosses for days to $50 \%$ flowering and days to maturity.

In case of plant height, best signicant and positive standard heterosis results were reported by RH-749 x RB-50 (17.95\%), Vardan x RL-1359 (17.18\%) and Vardan x RGN-145 (16.22\%). For number of primary branches per plant and 100-seed weight, Vardan x Kranti reported best standard heterosis of $47.92 \%$ and $24.19 \%$ respectively, followed by Vardan x RGN-145 (47.73\%) for number of primary branches per plant and Vardan x RL-1359 for 100-seed weight; showing the superior performance of Vardan for various characters. 
Table.1.2 Estimates of standard heterosis over check (RGN-13) on pooled data basis

\begin{tabular}{|c|c|c|c|c|c|c|c|c|c|c|c|c|c|}
\hline S.N. & Hybrids & $\begin{array}{l}\text { Days to } \\
50 \% \\
\text { Flowering }\end{array}$ & $\begin{array}{l}\text { Days to } \\
\text { Maturity }\end{array}$ & $\begin{array}{c}\text { Plant } \\
\text { height } \\
(\mathrm{cm})\end{array}$ & $\begin{array}{c}\text { No. of } \\
\text { Primary } \\
\text { branches per } \\
\text { plant }\end{array}$ & $\begin{array}{l}\text { No. of } \\
\text { siliqua per } \\
\text { plant }\end{array}$ & $\begin{array}{l}\text { Siliqua } \\
\text { length } \\
\text { (cm) }\end{array}$ & $\begin{array}{c}\text { No. of } \\
\text { seeds per } \\
\text { siliqua }\end{array}$ & $\begin{array}{c}\text { 100- seed } \\
\text { weight } \\
(\mathrm{gm})\end{array}$ & $\begin{array}{l}\text { Stover } \\
\text { yield per } \\
\text { plant } \\
\text { (gm) }\end{array}$ & $\begin{array}{l}\text { Seed Yield } \\
\text { per plant } \\
\text { (gm) }\end{array}$ & $\begin{array}{c}\text { Harvest } \\
\text { Index } \\
(\%)\end{array}$ & $\begin{array}{c}\text { Oil } \\
\text { Content } \\
(\%)\end{array}$ \\
\hline 1 & RGN13 x RGN145 & $3.14 *$ & $1.70 *$ & -4.63 & 19.59 & $47.50 *$ & 0.97 & -13.44 & -3.43 & 6.54 & 23.23 & 5.55 & -0.56 \\
\hline 3 & RGN13 x RL-1359 & $3.14 *$ & 0.95 & -0.37 & 1.11 & 23.99 & -2.95 & $-19.25^{*}$ & -7.06 & -21.72 & -3.54 & $13.58^{*}$ & 0.55 \\
\hline 6 & RGN73 x RGN145 & 2.32 & $1.64 *$ & 10.19 & $31.48^{*}$ & $43.28 *$ & 3.30 & 7.84 & -1.21 & 18.28 & $40.59 *$ & $12.77^{*}$ & -3.57 \\
\hline 7 & RGN73 x Kranti & $-3.42 * *$ & $-1.84 *$ & 13.75 & $30.14 *$ & $73.84 * *$ & $18.17 *$ & $17.29 *$ & $20.77 * *$ & $46.65^{*}$ & $69.65 * *$ & 9.61 & -3.49 \\
\hline 8 & RGN73 x RL-1359 & $5.19 * *$ & $2.19 * *$ & $14.37 *$ & 21.71 & $62.38 * *$ & 10.35 & 8.68 & 6.85 & 19.47 & 34.07 & 7.74 & $-9.91 * *$ \\
\hline 9 & RGN73 x Geeta & $3.41 * *$ & 0.95 & 2.64 & 29.95 & 32.50 & 4.56 & 5.23 & 4.23 & 4.13 & 15.61 & 8.03 & -0.29 \\
\hline 10 & RGN73 x RB-50 & $3.27 *$ & 0.21 & 1.10 & 20.77 & 14.56 & 7.16 & 1.76 & 10.48 & 30.23 & 22.19 & -6.02 & -2.23 \\
\hline 11 & RGN $48 \times$ RGN145 & $-4.24 * *$ & $-3.46^{* *}$ & 7.24 & 19.57 & 25.74 & 9.61 & 0.62 & 4.84 & 3.17 & 24.63 & 8.88 & -1.88 \\
\hline 13 & RGN $48 \times$ RL-1359 & -0.41 & -0.29 & 2.84 & 6.60 & 4.42 & 7.87 & $-16.4^{*}$ & -7.66 & -18.93 & -12.44 & 4.31 & -1.63 \\
\hline 14 & RGN 48 x Geeta & 0.68 & 0.08 & 5.30 & 11.66 & 10.68 & 1.08 & $-15.58 *$ & -1.21 & -13.36 & -5.15 & 3.82 & -3.53 \\
\hline 16 & RGN 229 x RGN145 & $-4.14 * *$ & $-1.72 *$ & 3.01 & 0.19 & 7.88 & -4.34 & -12.79 & -3.43 & -22.06 & -11.53 & 10.13 & -2.09 \\
\hline 17 & RGN 229 x Kranti & $-7.15^{* *}$ & $-5.26 * *$ & 4.58 & 18.49 & $62.09 * *$ & 13.50 & 2.76 & 9.48 & 19.91 & 16.34 & -3.31 & 1.32 \\
\hline 18 & RGN $229 \times$ RL-1359 & $-3.96 * *$ & $-2.50 * *$ & 4.68 & 13.53 & $58.98 * *$ & -1.60 & -7.62 & $13.1 *$ & 0.41 & 13.94 & 4.87 & 1.07 \\
\hline 19 & RGN 229 x Geeta & -2.28 & $-2.15 * *$ & -0.92 & 8.23 & $47.12 *$ & -1.82 & -11.08 & $-12.1^{*}$ & 7.55 & 8.37 & -2.04 & -4.01 \\
\hline 20 & RGN 229 x RB-50 & $-4.60 * *$ & $-3.08 * *$ & -0.02 & 1.73 & 12.64 & 9.59 & 1.93 & 5.85 & -20.01 & -10.54 & 8.89 & 0.20 \\
\hline 21 & RGN 298 x RGN145 & -0.55 & -1.41 & 9.30 & 27.62 & 34.01 & 12.32 & 6.09 & 0.81 & 15.88 & $42.23 *$ & 10.87 & -2.73 \\
\hline 22 & RGN 298 x Kranti & $-4.24 * *$ & $-3.27 * *$ & 3.35 & 25.96 & 34.13 & 12.11 & 7.42 & 2.82 & 11.08 & $40.91 *$ & $16.19 * *$ & -1.48 \\
\hline 23 & RGN $298 \times$ RL-1359 & $-2.87 *$ & $-2.21 * *$ & 5.82 & 11.66 & 25.21 & 13.90 & -0.26 & 5.04 & 4.05 & 30.68 & $16.61 * *$ & -1.20 \\
\hline 24 & RGN 298 x Geeta & $-3.15^{*}$ & $-2.83 * *$ & 12.71 & 29.69 & $42.00 *$ & 5.73 & 4.73 & -2.42 & 7.05 & 27.04 & 8.91 & $-5.07 *$ \\
\hline 25 & RGN $298 \times$ RB-50 & $-5.06 * *$ & $-3.15 * *$ & 4.20 & 28.86 & 24.41 & $14.60 *$ & 9.55 & -4.03 & 5.35 & 26.29 & 7.95 & 1.25 \\
\hline 26 & RGN 303 x RGN145 & $2.59 *$ & 0.70 & 3.42 & 11.99 & 13.68 & 0.91 & -5.87 & $-14.11 *$ & -21.49 & -8.56 & 9.70 & -2.15 \\
\hline 27 & RGN 303 x Kranti & 0.27 & -0.08 & 4.03 & 9.37 & 14.59 & 0.58 & 4.02 & -8.06 & -8.29 & 9.21 & $13.62 *$ & -2.91 \\
\hline 28 & RGN 303 x RL-1359 & 1.91 & -0.23 & 3.72 & 9.67 & 24.15 & 5.16 & 0.71 & $-13.51 *$ & -11.01 & -6.90 & 0.81 & 3.32 \\
\hline 29 & RGN 303 x Geeta & $3.14 *$ & 0.33 & 2.47 & 3.30 & 20.82 & 2.26 & 4.83 & $-14.52 *$ & -21.99 & -11.61 & 10.34 & 1.57 \\
\hline 30 & RGN 303 x RB-50 & 0.54 & 0.41 & 7.49 & 0.42 & 16.46 & 1.84 & -11.69 & -10.28 & -6.11 & -11.12 & -0.46 & -0.62 \\
\hline 31 & Bio 902 x RGN145 & $3.96 * *$ & 0.95 & 0.13 & 4.53 & -9.74 & -1.97 & -2.40 & -1.61 & -5.78 & -3.95 & 1.81 & $-5.89^{*}$ \\
\hline 32 & Bio 902 x Kranti & $-3.55 * *$ & $-3.27 * *$ & 8.15 & 22.12 & 33.26 & $16.86^{*}$ & 3.24 & 4.64 & 10.65 & 32.60 & 11.11 & -2.07 \\
\hline 33 & Bio $902 \times$ RL-1359 & 1.77 & 0.46 & 4.45 & 15.75 & -18.54 & 3.28 & -9.82 & -8.67 & -10.49 & -12.95 & -1.53 & $-6.92 * *$ \\
\hline 34 & Bio 902 x Geeta & $3.00^{*}$ & 1.08 & -2.30 & 17.28 & -7.81 & -1.07 & -0.77 & -8.87 & -14.04 & -17.45 & -1.78 & -4.77 \\
\hline 35 & Bio 902 x RB-50 & $3.82 * *$ & $2.13 * *$ & 0.45 & 0.10 & -3.30 & -2.44 & $-15.70^{*}$ & -10.48 & -10.4 & 4.83 & $13.02 *$ & $-7.44 * *$ \\
\hline 36 & RGN $236 \times$ RGN145 & $-7.93 * *$ & $-6.13 * *$ & 1.70 & 18.84 & 1.59 & -7.33 & -5.09 & -10.28 & -0.11 & 1.89 & -2.02 & -0.21 \\
\hline 37 & RGN 236 x Kranti & $-7.69 * *$ & $-5.75^{* *}$ & 2.04 & 16.78 & 23.55 & 1.26 & 11.53 & 1.01 & 18.34 & 15.87 & -2.47 & -2.89 \\
\hline 38 & RGN $236 \times$ RL-1359 & $-7.60 * *$ & $-5.19 * *$ & -0.74 & 4.30 & -13.24 & 7.57 & -8.34 & -6.05 & 14.93 & 6.37 & -3.22 & -3.56 \\
\hline
\end{tabular}




\begin{tabular}{|c|c|c|c|c|c|c|c|c|c|c|c|c|c|}
\hline 39 & RGN 236 x Geeta & $-7.24 * *$ & $-5.32 * *$ & 0.15 & 14.95 & 11.95 & 2.70 & -5.05 & 2.62 & -14.45 & -13.47 & 4.70 & -3.24 \\
\hline 40 & RGN $236 \times$ RB-50 & $-7.88 * *$ & $-5.75 * *$ & 8.30 & 2.64 & 33.00 & 3.52 & 1.75 & $-15.32 * *$ & 19.04 & 8.56 & -7.81 & -3.39 \\
\hline 41 & Rohini x RGN145 & $-2.73 *$ & -1.28 & 0.06 & 12.31 & -15.64 & -0.39 & -4.82 & $-16.53 * *$ & -18.77 & -20.54 & 3.20 & 0.23 \\
\hline 42 & Rohini x Kranti & 2.32 & 1.20 & -2.28 & 10.89 & -26.4 & 1.57 & -7.13 & $-18.55 * *$ & -20.55 & -25.30 & -3.33 & -3.57 \\
\hline 43 & Rohini x RL-1359 & -0.69 & -0.54 & -4.52 & 16.96 & -34.07 & 4.07 & -6.19 & 0.60 & -2.19 & -4.04 & 1.14 & $-5.04 *$ \\
\hline 44 & Rohini x Geeta & 0.82 & 0.14 & -0.63 & 5.72 & -3.65 & 2.11 & -2.52 & -8.87 & -12.80 & -12.49 & -0.63 & $-6.20 *$ \\
\hline 45 & Rohini x RB-50 & 0.82 & -0.54 & 10.71 & 7.14 & 10.76 & 6.41 & -9.26 & $-14.72 *$ & -12.80 & -15.09 & 0.86 & -4.55 \\
\hline 46 & GM 3 x RGN145 & -0.69 & -0.97 & 1.64 & 9.68 & 0.99 & 0.01 & 5.11 & $11.69^{*}$ & -3.99 & -12.83 & -4.34 & $-10.79 * *$ \\
\hline 47 & GM 3 x Kranti & $-4.10 * *$ & $-1.78 *$ & -5.54 & 7.93 & 23.48 & 11.31 & -0.16 & -2.82 & -8.47 & -15.74 & -5.16 & $-10.52 * *$ \\
\hline 48 & GM 3 x RL-1359 & $-2.73 *$ & $-2.09 * *$ & -3.38 & 10.43 & 17.43 & 0.91 & -4.27 & -0.60 & -11.09 & -21.08 & -6.01 & $-10.33 * *$ \\
\hline 52 & RN 393 x Kranti & $2.59 *$ & 0.83 & -0.35 & 8.34 & 12.18 & 2.72 & 1.55 & $-14.31 *$ & -14.16 & -26.51 & -10.26 & -2.42 \\
\hline 53 & RN 393 x RL-1359 & -1.78 & -1.18 & 7.8 & 13.64 & 14.37 & $14.96^{*}$ & -5.32 & -1.81 & -11.69 & -19.21 & -4.62 & -4.08 \\
\hline 54 & RN 393 x Geeta & $-2.73 *$ & $-1.59 *$ & 4.41 & 16.41 & $49.68 * *$ & -0.15 & -3.35 & $15.32 * *$ & -3.49 & -12.17 & -8.13 & -4.32 \\
\hline 55 & RN 393 x RB-50 & $3.27 *$ & 1.28 & 2.56 & 24.01 & $65.90 * *$ & 3.34 & -1.64 & 4.03 & -1.53 & -9.65 & -5.46 & -1.02 \\
\hline 56 & CS $52 \times$ RGN145 & -0.82 & -0.79 & -2.4 & -3.76 & 24.01 & 4.43 & -7.07 & $-12.7^{*}$ & -13.39 & -24.82 & -10.91 & $-9.09 * *$ \\
\hline 57 & CS 52 x Kranti & -2.05 & $-1.65^{*}$ & -1.2 & 15.49 & 24.32 & 8.58 & -8.11 & $-12.1^{*}$ & -18.80 & -25.71 & -3.50 & $-5.68 *$ \\
\hline 58 & CS x RL-1359 & -2.05 & -1.10 & -2.55 & 6.38 & 9.91 & 7.84 & 2.15 & -10.69 & -6.39 & -16.78 & -7.46 & $-7.5 * *$ \\
\hline 59 & CS 52 x Geeta & $-4.78 * *$ & $-2.59 * *$ & -3.16 & 8.01 & 11.25 & -3.33 & -1.36 & $-12.7 *$ & -18.05 & -25.34 & -3.82 & $-6.52 *$ \\
\hline 60 & CS $52 \times$ RB-50 & $-2.73 *$ & $-1.97 * *$ & -3.25 & 2.08 & 16.52 & -2.35 & -7.07 & $-12.3^{*}$ & -23.56 & -27.02 & 2.30 & -4.42 \\
\hline 61 & Pusa Bold x RGN145 & -0.82 & -0.66 & 3.24 & 5.92 & 4.13 & 2.01 & 0.22 & -1.81 & -14.60 & -11.28 & 1.63 & $-9.86 * *$ \\
\hline 62 & Pusa Bold x Kranti & 0.95 & 0.15 & 0.76 & 10.78 & 13.36 & 2.84 & -0.76 & -8.47 & 0.72 & 4.46 & 0.54 & $-6.62 * *$ \\
\hline 64 & Pusa Bold x Geeta & 1.09 & 0.06 & 1.67 & 14.75 & 17.96 & 0.69 & -0.71 & -5.24 & -5.16 & -0.60 & 2.62 & $-6.68 * *$ \\
\hline 65 & Pusa Bold x RB-50 & $3.55 * *$ & 1.14 & 0.85 & 3.20 & -18.81 & -3.04 & 4.83 & $-13.17 *$ & -16.72 & -8.12 & 5.49 & $-10.26 * *$ \\
\hline 66 & Laxmi x RGN145 & -2.19 & $-2.15 * *$ & 7.71 & 23.61 & $51.28 * *$ & 12.77 & 11.14 & 6.45 & 32.34 & $49.14 *$ & 4.93 & $-6.42 *$ \\
\hline 67 & Laxmi x Kranti & -1.37 & -1.10 & -3.44 & 18.84 & 32.26 & 8.23 & 12.58 & -3.63 & -12.78 & -15.03 & -3.17 & $-6.74 * *$ \\
\hline 68 & Laxmi x RL-1359 & -2.19 & $-1.74 *$ & 3.2 & 5.26 & -6.03 & 2.48 & -2.29 & -10.48 & -4.87 & -9.00 & -1.00 & $-5.8^{*}$ \\
\hline 69 & Laxmi x Geeta & 1.09 & -0.04 & -1.16 & 8.70 & -11.15 & 1.92 & -6.81 & 2.82 & 2.34 & -4.40 & -6.07 & $-5.26^{*}$ \\
\hline 71 & RH $749 \times$ RGN145 & $-5.88 * *$ & $-2.9 * *$ & 0.93 & 29.94 & 25.44 & $17.43^{*}$ & 9.05 & 9.07 & 26.07 & 27.36 & -0.17 & -3.00 \\
\hline 72 & RH 749 x Kranti & 1.77 & 0.52 & 1.56 & 20.90 & $37.44 *$ & 2.48 & -8.94 & -1.81 & 5.22 & 11.44 & 1.33 & -2.88 \\
\hline 75 & RH $749 \times$ RB-50 & $-9.25 * *$ & $-5.85 * *$ & $17.95^{*}$ & 23.79 & $102.68 * *$ & 8.65 & 11.75 & $16.73 * *$ & $52.80 *$ & $82.92 * *$ & $13.06^{*}$ & -1.31 \\
\hline 77 & NPJ 113 x Kranti & $-4.51 * *$ & $-2.21 * *$ & 0.01 & 19.48 & $52.85 * *$ & 2.84 & 0.77 & -5.24 & -7.78 & 3.23 & 6.20 & -5.02 \\
\hline 78 & NPJ 113 x RL-1359 & $-2.60 *$ & -1.03 & -1.90 & 14.65 & 28.08 & 6.59 & 0.66 & $-12.3^{*}$ & 4.19 & -7.98 & -8.25 & $-8.76 * *$ \\
\hline 79 & NPJ 113 x Geeta & $-3.28 *$ & -0.97 & 2.21 & 15.09 & 21.94 & -6.04 & -7.40 & 3.23 & -18.92 & -14.45 & 11.34 & -3.95 \\
\hline 80 & NPJ 113 x RB-50 & $-5.74 * *$ & $-2.40 * *$ & 3.01 & 19.72 & 19.06 & 12.09 & 9.71 & -10.48 & -10.03 & -11.82 & -0.05 & -1.95 \\
\hline 81 & PBR 357 x RGN145 & 1.77 & 1.26 & 0.07 & 9.35 & $38.82 *$ & 5.52 & -6.47 & $15.93 * *$ & 16.49 & 26.89 & 6.27 & $-5.08^{*}$ \\
\hline 82 & PBR 357 x Kranti & $3.82 * *$ & $1.70 *$ & -1.97 & 15.73 & 31.16 & 4.07 & -2.57 & $-14.31 *$ & 11.36 & 12.24 & 0.20 & -2.90 \\
\hline 83 & PBR 357 x RL-1359 & $4.78^{* *}$ & $1.70 *$ & 0.57 & 7.71 & $41.69 *$ & 5.67 & -3.29 & -9.07 & -2.96 & -0.06 & 4.95 & $-5.05^{*}$ \\
\hline 84 & PBR 357 x Geeta & $3.14^{*}$ & 0.70 & 2.82 & 15.20 & 29.81 & -0.23 & 2.53 & -3.23 & -17.62 & -15.76 & 5.51 & -3.69 \\
\hline 86 & NRCDR $2 \times$ RGN145 & -0.14 & -0.54 & 1.17 & 8.23 & 19.63 & 11.75 & -3.84 & $-16.94 * *$ & -2.73 & -13.22 & -8.20 & -2.87 \\
\hline
\end{tabular}




\begin{tabular}{|c|c|c|c|c|c|c|c|c|c|c|c|c|c|}
\hline 87 & NRCDR 2 x Kranti & -1.37 & -0.41 & 9.38 & 7.70 & $50.32 * *$ & 6.69 & -4.17 & 8.06 & -1.82 & 0.70 & 1.43 & -2.33 \\
\hline 88 & NRCDR $2 \times$ RL-1359 & $2.87 *$ & 0.77 & 5.73 & 5.84 & $43.49 *$ & 2.80 & -3.06 & -9.68 & -7.99 & -2.93 & 4.86 & 2.05 \\
\hline 90 & NRCDR 2 x RB-50 & 1.77 & 0.46 & 5.89 & 24.56 & $57.35 * *$ & 9.75 & 6.43 & $-14.31 *$ & 26.93 & 10.92 & -10.28 & -3.75 \\
\hline 91 & PBR 378 x RGN145 & $2.73 *$ & 0.70 & 4.77 & 14.43 & $48.24 * *$ & 2.16 & 0.55 & 5.24 & 19.3 & 17.98 & -3.11 & -3.92 \\
\hline 92 & PBR 378 x Kranti & $5.46 * *$ & $1.82 *$ & 0.89 & -0.45 & $64.29 * *$ & -0.65 & -0.81 & 0.2 & -12.55 & -5.55 & 6.25 & -2.05 \\
\hline 93 & PBR 378 x RL-1359 & 1.91 & 0.39 & 1.13 & 9.44 & $55.66 * *$ & 3.12 & 0.60 & -0.2 & -9.99 & -20.31 & -8.41 & -1.97 \\
\hline 94 & PBR 378 x Geeta & 2.05 & 0.64 & 2.29 & 7.35 & $42.75^{*}$ & -4.83 & 0.55 & -3.63 & -1.04 & -4.24 & 2.32 & -1.99 \\
\hline 96 & KDM-10-49 x RGN145 & -2.32 & -1.16 & 3.49 & 0.76 & 19.89 & 0.49 & -7.84 & -7.06 & -13.19 & -10.21 & 5.23 & $-6.13 *$ \\
\hline 97 & KDM-10-49x Kranti & $-3.14 *$ & $-1.97 * *$ & -3.98 & 6.58 & 14.71 & 1.37 & -8.23 & 2.42 & -17.89 & -16.47 & 5.01 & -2.58 \\
\hline 98 & KDM-10-49 x RL-1359 & 0.00 & -0.41 & -2.66 & 0.19 & 32.22 & -5.89 & -3.89 & 10.28 & -12.93 & -3.36 & 7.24 & -3.16 \\
\hline 99 & KDM-10-49 x Geeta & $-3.69 * *$ & $-1.9 * *$ & -2.48 & 2.54 & $41.33^{*}$ & -4.95 & -11.36 & -9.27 & -4.81 & -4.38 & 0.55 & -3.33 \\
\hline 100 & KDM-10-49x RB-50 & $-3.14 *$ & $-1.47 *$ & 0.24 & 1.62 & $39.12 *$ & 4.67 & 1.21 & -9.48 & -9.62 & -2.96 & 8.74 & -1.81 \\
\hline 101 & RLM $619 \times$ RGN145 & 2.46 & 0.58 & 2.90 & 14.97 & $42.85^{*}$ & -1.99 & 6.32 & $-17.54 * *$ & 5.10 & 1.00 & -3.93 & -4.05 \\
\hline 102 & RLM 619 x Kranti & 1.91 & 0.66 & 0.27 & 6.05 & 18.83 & -2.99 & -3.50 & -8.06 & 8.55 & 11.73 & 2.34 & $-6.80 * *$ \\
\hline 103 & RLM $619 \times$ RL-1359 & -0.69 & -0.48 & 1.89 & 6.14 & $50.71 * *$ & 2.73 & -3.89 & $-15.52 * *$ & -0.94 & -6.19 & -2.99 & $-10.62 * *$ \\
\hline 104 & RLM 619 x Geeta & 0.41 & -0.41 & -2.18 & 11.66 & 29.03 & 6.02 & -10.81 & -11.49 & -5.94 & 1.92 & 4.83 & $-7.63 * *$ \\
\hline 105 & RLM 619x RB-50 & -2.32 & -1.41 & -0.65 & 5.05 & 25.69 & -5.79 & 6.04 & -1.81 & -7.72 & -1.16 & 6.35 & $-7.05 * *$ \\
\hline 106 & Vardan x RGN145 & 1.64 & 1.01 & $16.22 *$ & $47.73 * *$ & $85.88 * *$ & $27.15^{* *}$ & 10.68 & $17.54 * *$ & $53.26 * *$ & $67.45 * *$ & 6.53 & -2.40 \\
\hline 107 & Vardan x Kranti & $-4.87 * *$ & $-2.21 * *$ & $15.21 *$ & $47.92 * *$ & $60.06 * *$ & $24.02 * *$ & 10.81 & $24.19 * *$ & $51.48^{*}$ & $79.32 * *$ & $12.60^{*}$ & -3.29 \\
\hline 108 & Vardan x RL-1359 & 1.64 & 0.64 & $17.18^{*}$ & $36.24 *$ & $58.49 * *$ & 11.75 & $15.65^{*}$ & $22.38 * *$ & $46.77 *$ & $68.65 * *$ & 9.25 & -3.40 \\
\hline 109 & Vardan x Geeta & 0.68 & -0.10 & 9.39 & $46.01 * *$ & $61.60 * *$ & 7.62 & $17.30^{*}$ & 9.48 & $47.63^{*}$ & $55.27 * *$ & 0.55 & -2.15 \\
\hline 110 & Vardan x RB-50 & $-5.60 * *$ & $-3.70 * *$ & 11.39 & $42.93 * *$ & $56.55 * *$ & $14.21 *$ & $18.18^{*}$ & 7.66 & $51.92 *$ & $61.58 * *$ & 2.74 & 0.00 \\
\hline 111 & PCR 7 x RGN145 & -0.28 & -0.29 & -1.42 & 1.64 & $42.10^{*}$ & -6.68 & -2.30 & 10.28 & -8.30 & -7.14 & 3.34 & -2.37 \\
\hline 112 & PCR 7 x Kranti & 0.41 & 0.08 & -3.62 & 8.78 & 33.40 & -3.29 & -4.11 & 3.63 & -13.59 & -3.51 & $14.48^{*}$ & -2.58 \\
\hline 113 & PCR 7 x RL-1359 & -0.14 & -0.48 & -2.07 & 2.06 & 22.67 & 0.83 & -7.08 & 0.20 & -26.53 & -20.00 & 7.14 & $-11.61 * *$ \\
\hline 114 & PCR 7 x Geeta & 0.82 & -0.66 & 1.11 & 1.55 & 26.40 & -1.14 & 1.00 & -7.86 & -17.20 & -10.59 & 5.22 & $-7.66 * *$ \\
\hline 115 & PCR 7 x RB-50 & 0.13 & -0.17 & 3.03 & -2.01 & 28.42 & 1.42 & -2.95 & $-16.13 * *$ & -4.15 & -2.01 & 2.58 & $-6.49 *$ \\
\hline 116 & JMWR-08-3 x RGN145 & $-3.28 *$ & $-2.46^{* *}$ & 3.92 & -2.96 & 26.09 & 1.13 & -7.73 & $-12.50 *$ & -3.35 & -5.98 & -5.33 & $-6.37 *$ \\
\hline 117 & JMWR-08-3 x Kranti & $-3.14 *$ & $-2.09 * *$ & 3.42 & -4.74 & 22.34 & 3.86 & -11.19 & -9.68 & -1.29 & -5.86 & -4.35 & -4.17 \\
\hline 118 & JMWR-08-3XRL-1359 & $-2.60 *$ & $-2.15 * *$ & 0.70 & -7.15 & 20.83 & 2.43 & 1.60 & 6.85 & 9.03 & 3.35 & -5.57 & $-5.75^{*}$ \\
\hline 119 & JMWR-08-3 x Geeta & 0.27 & -0.47 & 2.14 & 10.56 & $58.18 * *$ & 2.46 & -5.59 & $-14.52 *$ & -1.51 & -7.83 & -6.33 & $-10.38 * *$ \\
\hline 120 & JMWR-08-3 x RB-50 & -0.82 & -0.54 & 2.73 & 17.17 & $37.15^{*}$ & 0.24 & -4.56 & -3.23 & -6.26 & -7.53 & -1.73 & $-7.34 * *$ \\
\hline 121 & RH-30 x RGN145 & -2.01 & $-1.84 *$ & 8.39 & 23.45 & $47.57 *$ & 11.24 & 9.08 & 10.89 & $45.33 *$ & $45.72 *$ & -1.61 & -1.34 \\
\hline 124 & RH-30 x Geeta & $-4.64 * *$ & $-3.08 * *$ & 6.18 & 11.31 & 25.89 & 10.30 & 7.14 & 9.27 & 25.52 & 30.73 & -2.24 & -1.62 \\
\hline 125 & RH-30 x RB-50 & 0.13 & -0.35 & 2.08 & 8.93 & 18.54 & 2.26 & 7.53 & $13.71 *$ & 14.31 & 13.33 & -3.27 & -2.06 \\
\hline
\end{tabular}


Similarly, Vardan $\mathrm{x}$ RGN-145 reported maximum heterosis for siliqua length through its performance of $27.15 \%$, showing a significant increase over the check followed by Vardan x Kranti (24.02\%) and RGN-73 x Kranti (18.17\%).

Highest percentage of improvement for number of siliqua per plant over the check variety was reported by RH-749 x RB-50 (102.68\%) followed by Vardan x RGN -145 (85.88\%) and RGN-73 x Kranti (73.84\%). For number of seeds per siliqua, moderate standard heterosis had been reported by various crosses namely, Vardan x RB-50 (18.18\%), Vardan x Geeta (17.30\%) and RGN-73 x Kranti (17.29\%).

In case of seed yield per plant, RH-749 x RB50 reported the highest standard heterosis of $82.92 \%$ followed by Vardan X Kranti (79.32\%) and RGN 73 x Kranti ( 69.65\%). None of the hybrids were found to be showing significantly positive heterosis for harvest index and oil content. Similarly, For harvest index, low levels of non significant heterosis to moderate amounts of significantly positive heterosis were reported. RGN-298 x RL-1359 (16.61\%), RGN-298 x Kranti (16.19\%) and PCR-7 x Kranti (14.48\%) reported best performances for harvest index. A small number of crosses reported non significant but positive standard heterosis for oil content viz. RGN-303 x RL-1359 (3.32\%), RGN-303 x RB-50 (1.57\%) and NRCDR-2 x RL-1359 (2.05\%).

In the present study, standard heterosis has been estimated over the best check variety RGN-13. From the performance point of view a few crosses have reported significant standard heterosis for many characters. RGN $73 \mathrm{x}$ Kranti has reported heterosis for most of the traits except plant height, harvest index and oil content; Vardan $\mathrm{x}$ Kranti found superiority for all the traits except for number of seeds per siliqua, harvest index and oil content; Vardan x RB-50 reported significant heterotic effects for all the characters but not for plant height, 100-seed weight, harvest index and oil content and; RH-749 x RB-50 was yet another hybrid with the exception of number of primary branches per plant, siliqua length, harvest index and oil content found superiority for all the traits.

The maximum values of standard heterosis recorded were $82.92 \%$ for seed yield per plant while for yield components it was $53.26 \%$ for stover yield; $47.92 \%$ for number of primary branches per plant; $27.15 \%$ for siliqua length; $102.68 \%$ for number of siliquae per plant; $18.18 \%$ for number of seeds per siliqua; $24.19 \%$ for 100 -seed weight; $-6.13 \%$ for days to maturity; $-9.25 \%$ for days to $50 \%$ flowering; and $17.95 \%$ for plant height.

In matters of performance for different traits various crosses were found to be giving top performances viz, RH-749 x RB-50, RGN$236 \times$ RGN-145 and RGN-236 x RB-50 have been identified as highly heterotic crosses for days to $50 \%$ flowering and days to maturity. Similar results were reported by Gupta, Chaudhary and Lal (2010) and Monpara and Dobariya (2007) for days to 50\% flowering and days to maturity from their studies on Indian mustard.

In case of plant height, for which tallness has been considered as a desirable feature, best signicant and positive standard heterosis results were reported by RH-749 x RB-50 (17.95\%), Vardan x RL-1359 (17.18\%) and Vardan x RGN-145 (16.22\%). These findings corroborates with the results of Singh, Kumar and Singh (2012) and Meena etal.(2014).

For number of primary branches per plant and 100-seed weight, Vardan x Kranti reported best standard heterosis of $47.92 \%$ and $24.19 \%$ respectively followed by Vardan x RGN-145 
(47.73) for number of primary branches per plant and Vardan x RL-1359 for 100-seed weight; showing the superior performance of Vardan for various characters. Findings of similar nature was reported by Gupta, Chaudhary and Lal (2010) for number of primary branches per plant and seed weight and, for seed weight by Tyagi et al., (2000) and Monpara and Dobariya (2007)

Vardan $\mathrm{x}$ RGN-145 reported maximum heterosis for siliqua length through their performance of $27.15 \%$, showing a significant increase over the best check followed by Vardan x Kranti (24.02\%) and RGN-73 x Kranti (18.17\%). Similar findings were reported by Teklewood and Becker (2005) and Monpara and Dobariya (2007). Highest percentage of improvement in performance for number of siliqua per plant over the best check variety was reported by RH-749 x RB$50(102.68 \%)$ followed by Vardan x RGN $145(85.88 \%)$ and RGN-73 x Kranti (73.84\%) showing the superiority of these crosses for this character. These findings are similar to the one reported by the Muhammad (2003) and Aher et al., (2009).

For number of seeds per siliqua, moderate standard heterosis have been reported by various crosses namely, Vardan x RB-50 $(18.18 \%)$, Vardan $x$ Geeta $(17.30 \%)$ and RGN-73 x Kranti (17.29\%). These findings have also been substantiated by the findings of Tyagi et al., (2000) and Prajapati et al., (2007) as they have also found positive heterosis for number of seeds per siliquae which directly contributes to the positive heterosis for seed yield per plant. In case of seed yield per plant, RH-749 x RB-50 reported the highest standard heterosis of $82.92 \%$ followed by Vardan X Kranti (79.32\%) and RGN 73 x Kranti ( 69.65\%). None of the hybrids were found to be showing significantly positive heterosis for the traits harvest index and oil content.
Similar to the our findings, moderate to high heterosis and significantly positive results for seed yield have also been reported by Prajapati et al., (2007), Tomar et al., (2014) and Aher et al., (2009).

For harvest index, low levels of non significant heterosis to moderate amounts of significantly positive heterosis were reported. RGN-298 x RL-1359 (16.61\%), RGN-298 x Kranti (16.19\%) and PCR-7 x Kranti (14.48\%) reported best performances for harvest index.

These findings have been substantiated by the findings of Prajapati et al., (2007) and Tyagi et al., (2000) since, they also reported moderate amount of positive hetrosis for harvest index. Standard heterosis results for oil content were found to be significantly negative for a few hybrids, where positively significant heterosis for oil content would have been a desirable feature. Nonetheless, a small number of crosses have reported non significant but positive standard heterosis for this trait viz. RGN-303 x RL-1359 (3.32\%), RGN-303 x RB-50 (1.57\%) and NRCDR-2 x RL-1359 (2.05\%). Results of this nature for oil content have also been reported by Prajapati et al., (2007), as low levels of non significant heterotic improvements for oil content were registered by them also.

In almost all the characters variable number of crosses depicted standard heterosis in both positive and negative direction, indicating that genes with negative as well as positive effects were dominant. This show of unpredictability highlights the role of non additive gene actions, which in turn may be due to dominance and/or epistasis.

In conclusion through the study it emerges that a few crosses reported significant standard heterosis for many characters. RGN $73 \mathrm{x}$ Kranti reported heterosis for most of the 
traits except for plant height, harvest index and oil content; Vardan $\mathrm{x}$ Kranti found superiority for all the traits except for number of seeds per siliqua, harvest index and oil content; Vardan x RB-50 reported significant heterotic effects for all the characters but not for plant height, 100-seed weight, harvest index and oil content and; RH-749 x RB-50 was yet another hybrid with the exception of number of primary branches per plant, siliqua length, harvest index and oil content found superiority for all the traits. These crosses can be utilized for further development of superior cultivars of Indian mustard.

\section{Acknowledgement}

Authors of this paper are thankful to the students and supervisors of Department of Plant Breeding and Genetics, SKRAUBikaner. Dr. K. S. Shekhawat for helping in estimating oil contents at their facilities in Dhanlaxmi Seeds, Himmatnagar, Gujarat.

\section{References}

Aher, C.D., Shelke, L.T., Chinchane, V.N., Borgaonkar, S.B., and Gaikwad, A.R. 2009. Heterosis for yield and yield components in Indian mustard [Brassica juncea (L.) Czern\&Coss.]. International Journal of Plant Sciences, 4(1): 30-32.

Anonymous 2015. FAO- Trade and Markets Division. Food Outlook .

Anonymous 2016. Oilseeds: World Markets and Trade. Foreign Agricultural Service, United States Department of Agriculture.

Gupta, P., Chaudhary, H.B., and Lal, S.K. 2010. Heterosis and combining ability analysis for yield and its components in Indian mustard [Brassica juncea (L.) Czern\&Coss.]. Frontiers of Agriculture in China, 4 (3): 299-307.

Meena, H.S., Ram, B., Kumar, A., Singh, B.K., Meena, P.D., Singh, V.V and Singh, D 2014. Heterobeltiosis and standard heterosis for seed yield and important traits in Brassica juncea. Journal of Oilseed Brassica, 5(2): 134-140.

Monpara, B. A. and Dobariya, K. L. 2007. Heterosis and combining ability in Indian mustard.J. Oilseeds Res., 24 (2): 306-308.

Prajapati, C. H., Patel, K. M., Patel, M. P. and Pathak H. C. 2007. Heterosis for seed yield and its components in Indian rapeseed,(Brassica campestris var. yellow sarson). J. Oilseeds Res., 24 (2): 309-310.

Singh, D.K., Kumar, K. and Singh, P. 2012. Heterosis and heritability analysis for different crosses in Brassica juncea with inheritance of white rust resistance. Journal of Oilseed Brassica, 3(1): 18-26.

Teklewold, A. and Becker, H. C. 2005. Heterosis and combining ability in a diallel cross of ethiopian mustard inbred lines. Crop Science, 34(1): 192- 196.

Tomar, A., Singh, M., Singh, S.K. and Yadav, R.K. 2014. Heterosis for seed yield and its component traits in Indian mustard [Brassica juncea (L.) Czern\&Coss.]. Current Advances in Agricultural Sciences, 6(2) : 183-185.

Tyagi, M.K., Chauhan, J.S., Yadav, S.K., Kumar, P.R. and Tyagi, P. 2000. Heterosis in intervarietal crosses in mustard [Brassica juncea (L.) Czern\&Coss.]. Annals of Biology, 16(2): 191-194.

\section{How to cite this article:}

Shekhawat, H. V. S., S. S. Shekhawat and Shekhawat, U. S. 2021. Heterosis Studies in Indian Mustard [Brassica juncea (L.) Czern \& Coss.] for Yield and its Related Attributes. Int.J.Curr.Microbiol.App.Sci. 10(01): 2268-2275. doi: https://doi.org/10.20546/ijcmas.2021.1001.262 